\title{
Evaluation of dermal thermal damage by multiphoton autofluorescence and second-harmonic-generation microscopy
}

\author{
Ming-Gu Lin* \\ Tsung-Lin Yang* \\ Cheng-Tien Chiang \\ Hsien-Ching Kao \\ Jin-Ning Lee \\ Wen Lo \\ National Taiwan University \\ Department of Physics \\ Taipei, Taiwan
}

\author{
Shiou-Hwa Jee \\ National Taiwan University Hospital and \\ National Taiwan University College \\ of Medicine \\ Department of Dermatology \\ Taipei, Taiwan
}

\section{Yang-Fang Chen}

Chen-Yuan Dong

National Taiwan University

Department of Physics

Taipei, Taiwan

\author{
Sung-Jan Lin \\ National Taiwan University Hospital and \\ National Taiwan University College \\ of Medicine \\ Department of Dermatology \\ Taipei, Taiwan
}

\begin{abstract}
We attempt to characterize the degree of skin thermal damage by using multiphoton microscopy to characterize dermal thermal damage. Our results show that dermal collagen and elastic fibers display different susceptibility to thermal injury. Morphologically, dermal collagen starts to denature at $60^{\circ} \mathrm{C}$ while fracture and aggregation of elastic fibers do not occur until $65^{\circ} \mathrm{C}$. With increasing temperatures, the structures of both elastic and collagen fibers deteriorate. While second-harmonic-generation (SHG) imaging is helpful in identifying the denaturation temperature of collagen, autofluorescence (AF) imaging can help to identify the structural alternations of tissue at higher temperatures when SHG signals have decayed. We also employ a ratiometric approach based on the AF-to-SHG index of dermis (ASID) to characterize the degree of dermal thermal damage. Use of the ASID index can bypass the difficulty in analyzing inhomogeneous dermal fibers and show that dermal collagen starts to denature at $60^{\circ} \mathrm{C}$. Our results suggest that with additional developments, multiphoton microscopy has potential to be developed into an effective in vivo imaging technique to monitor and characterize dermal thermal damage. ( ) 2006 Society of Photo-Optical Instrumentation Engineers. [DOI: 10.1117/1.2405347]

Keywords: multiphoton imaging; autofluorescence; second-harmonic generation; elastin; collagen; thermal damage.

Paper 06125R received May 18, 2006; revised manuscript received Aug. 21, 2006; accepted for publication Aug. 24, 2006; published online Dec. 28, 2006. This paper is a revision of a paper presented at the SPIE conference on Photonic Therapeutics and Diagnostics, Jan. 2006, San Jose, CA. The paper presented there appears (unrefereed) in SPIE Proceedings Vol. 6078A.
\end{abstract}

\section{Introduction}

The determination of the extent of thermal damage to skin has important clinical relevance in dermatology. In the case of burn victims, accurate assessment of the boundary of damaged tissues can help the clinicians in their removal and lead to recovery. In addition, thermal damage can be an unwanted side effect resulting from skin treatment procedures. Specifically, the use of lasers in skin rejuvenation, pigment treatment, hair removal, and treatment of vascular lesions can lead to the deposition of thermal energy outside the target tissue, causing unwanted thermal injuries. ${ }^{1-5}$ Other treatment modalities including intense pulse light and radiofrequency can also produce thermal damages. ${ }^{6,7}$ Unless an effective monitoring technique is developed, the identification of appropriate parameters to enhance treatment and minimize the unwanted thermal injury will continue to be based on trial-and-error practices. Therefore, the development of a minimally invasive

*These authors contributed equally to this work.

Address all correspondence to Chen-Yuan Dong, Tel.: 8862-2-3366-5155, Fax: 886-2-2363-9984, E-mail: cydong@phys.ntu.edu.tw; and Sung-Jan Lin, Tel: 886-2-23562141, Fax: 886-2-23934177, E-mail: sjlin@ha.mc.ntu.edu.tw technique capable of monitoring the extent of skin thermal damage is invaluable to clinical dermatology.

A number of techniques have proven to be useful in characterizing skin thermal damage. While histological analysis is in common use, ${ }^{1}$ other techniques such as reflected confocal microscopy, indocyanine green fluorescence imaging, optical coherence tomography (OCT), and laser Doppler scanning have been utilized to assess skin thermal damage. ${ }^{8-12}$ An alternative approach in assessing skin thermal damage is multiphoton microscopy. ${ }^{13}$ For the assessment of skin conditions, multiphoton microscopy offers several distinct advantages. First, imaging using the point-like excitation volume results in high-contrast images without confocal detection. ${ }^{14,15}$ In addition, the limited excitation volume reduces the overall specimen damage. Finally, the near-infrared excitation photons are absorbed and scattered less by tissue constituents and deeper sample imaging depths can be achieved. ${ }^{16,17}$

In the case of skin imaging, multiphoton microscopy offers another distinct advantage. The abundant collagen fibers in the dermis are capable of generating intense second-

1083-3668/2006/11(6)/064006/6/\$22.00 @ 2006 SPIE 


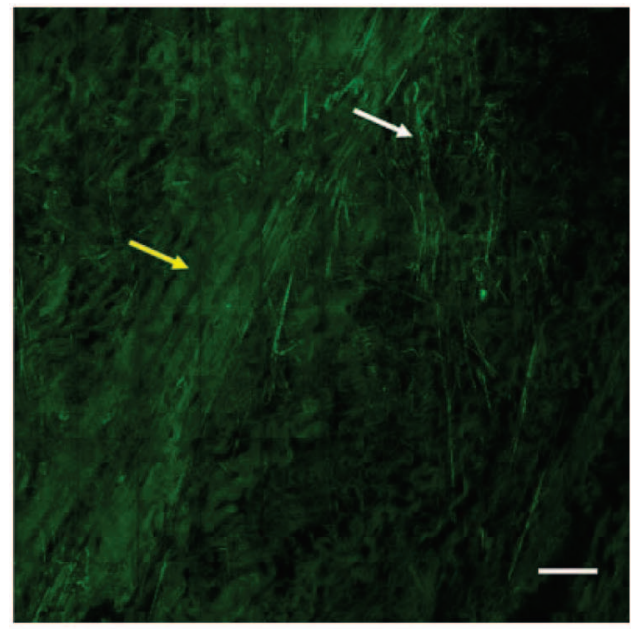

(a)

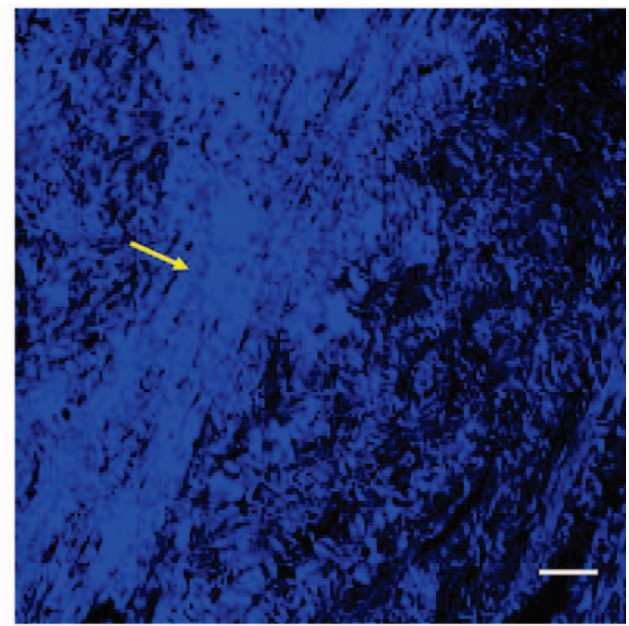

(b)

Fig. 1 Multiphoton (a) AF and (b) SHG images of foreskin dermis. SHG signals are blue and AF signals are green.

harmonic-generation (SHG) signals. ${ }^{14,18}$ While fluorescence emission typically occurs at longer wavelengths than the wavelength of the combined photons used in multiphoton excitation, SHG signal occurs at precisely half the wavelength of the excitation wavelength. ${ }^{19-23}$ Therefore, the clear spectral separation between the SHG collagen fibers and autofluorescent elastic fibers can be used to identify thermal changes associated with each tissue type and can potentially lead to a quantitative parameter characterizing the extent of thermal damage of the dermis. ${ }^{18,22,23}$ In this work, we used the combined imaging modality of multiphoton autofluorescence (AF) and SHG microscopy to image and characterize skin dermal damage. While the combination of forward and backward SHG has been proven to be useful in elucidating the SHG mechanism in rat tail tendons and the imaging of the cornea and sclera, ${ }^{24,25}$ we favor the use of backscattering geometry in SHG detection. Our choice is motivated by the clear advantage the backscattering approach offers in potential clinical applications.

\section{Materials and Methods}

The multiphoton microscope system used in this study is similar to the one previously described. ${ }^{18}$ A diode-pumped solid-state (DPSS) laser (Millennia X, Spectra Physics, Mountain View, Calif.) pumped titanium-sapphire laser system (Tsunami, Spectra Physics) was used as the excitation source. The 780-nm output of the laser system was guided toward a modified commercial upright microscope (E800, Nikon, Japan). Prior to entering the microscope, the excitation source was angularly deflected by an $\mathrm{x}-\mathrm{y}$ scanning system (Model 6220, Cambridge Technology, Cambridge, Mass). The input of the upright microscope was modified to accommodate a beam expander. The excitation source was beam expanded and reflected toward the focusing objective (Nikon $\mathrm{S}$ Fluor 40x, NA 1.30) by a primary dichroic (700DCSPXRUV$3 \mathrm{p}$, Chroma Technology, Rockingham, Vermont). The primary dichroic is a short-pass filter that transmits below the wavelength range of 350 to $700 \mathrm{~nm}$. The power at the sample was
$35 \mathrm{~mW}$. The nonlinear AF and SHG signal were generated at the sample focal plane and collected in the epi-illuminated or backscattering geometry by the same focusing objective. After passing through the primary dichroic, the AF and SHG signals were separated into two separate channels where they are detected by independent photomultiplier tubes (R7400P, Hamamatsu, Japan). The AF and SHG signals were separated by a secondary dichroic (435DCXR, Chroma Technology). The secondary dichroic is a long-pass filter that reflects below and transmits above $435 \mathrm{~nm}$. The SHG signal was further filtered by a SHG filter (HQ390/20, Chroma Technology) centered at $390 \mathrm{~nm}$ with a bandwidth of $20 \mathrm{~nm}$ while the AF was detected by a bandpass filter (E700sp-2P-E435lp, Chroma Technology) for broadband fluorescence detection between 435 and $700 \mathrm{~nm}$. To acquire a global image of the thermally treated specimens at high resolution, an $\mathrm{x}-\mathrm{y}$ sample positioning stage (H101, Prior Scientific, UK) was used to translate the skin specimen after each imaged frame. A $10 \times 10$ array of overlapping and individually beam scanned images, each with an area of $110 \times 110 \mu \mathrm{m}$,was then assembled.

Human foreskin specimens were used in this study. The human foreskin was cut into individual pieces and placed in a phosphate buffered saline (PBS) for $20 \mathrm{~min}$ at the desired temperature. Temperatures separated by $5^{\circ} \mathrm{C}$ intervals ranging from $25^{\circ} \mathrm{C}$ to $95^{\circ} \mathrm{C}$ were used. After the thermal treatment cycle, the specimens were removed, inverted, and mounted on slides for dermal imaging. Since the back side of the foreskin corresponds to the dermal layer, the foreskin is the ideal specimen for investigating dermal changes without histological preparation. In order to avoid surface artifacts, all images were acquired at approximate $30 \mu \mathrm{m}$ below the sample surface.

\section{Results}

Large-area multiphoton AF and SHG images of the skin are shown in Fig. 1. We observed interesting trends in SHG and $\mathrm{AF}$ within the skin dermis as a function of temperature. Con- 
sistent with our previous work, ${ }^{18}$ intensely autofluorescent elastic fibers can be observed in the skin [Fig. 1(a), white arrow]. On the other hand, we found that while the foreskin dermal collagen fibers are capable of generating intense SHG signals, they are also autofluorescent [Fig. 1(a), yellow arrow], although at lower level than the elastic fibers. In the isolated SHG image, collagen fibers are clearly visible and elastic fibers do not produce SHG signals [Fig. 1(b), yellow arrow].

For our study, we thermally treated the foreskin dermis at 15 different temperatures from $25^{\circ} \mathrm{C}$ to $90^{\circ} \mathrm{C}\left(5^{\circ} \mathrm{C}\right.$ intervals), and representative images of the combined $\mathrm{AF}$ and $\mathrm{SHG}$ data are shown in Fig. 2. We found that the appearance and organization of dermal fibers change as the temperature increases. First, as Fig. 2 shows, the general tendency is for the SHG intensity from dermal collagen to decrease with increasing temperatures. At a sufficiently high temperature of $60^{\circ} \mathrm{C}$, the fibrous structures of dermal collagen in SHG images start to be disrupted. Since SHG signals of collagen are sensitive to structural changes, ${ }^{22}$ the decrease of SHG indicates that the collagen fibers in the dermis begin to be disrupted at this temperature. As the temperature is further raised, the SHG signals further deteriorate and the presence of collagen fibers can not be easily visualized in the isolated SHG images. On the contrary, the AF of collagen fibers remains to be visible. Although the SHG intensity of collagen is progressively weakened above $60^{\circ} \mathrm{C}$, the $\mathrm{AF}$ signals can still be used to resolve collagen structures. From AF signals, dermal collagen preserves a fibrous structure at the temperatures lower than $60^{\circ} \mathrm{C}$. At $60^{\circ} \mathrm{C}$, the fibrous structures of collagen start to be disrupted. At $75^{\circ} \mathrm{C}$, a great proportion of collagen is molten into an amorphous structure. As the temperature is further raised, the collagen in the dermis appears to have become molten. This is consistent with the histological results showing a homogenized structure of collagen (data not shown). Hence, AF images can help to trace the presence of denatured collagen beyond the denaturation temperature. In addition, the AF images from our results also indicate that, after the disruption of structures responsible for SHG signals, collagen continues to undergo structural modifications at higher temperatures.

Compared with the collagen fibers, the elastic fibers appear to be less significantly affected by the thermal treatment. Morphologically, the elastic fibers still display a fine fibrous structure at $60^{\circ} \mathrm{C}$, the temperature at which collagen SHG signals start to fade. However, when the temperature is raised to $65^{\circ} \mathrm{C}$, an interesting result is revealed by the AF image. Autofluorescent elastic fibers start to fracture and become aggregated [Fig. 2(d), red arrow]. As temperature is further raised, the fibrous structures are progressively disrupted and the aggregations become more evident. We attempted to characterize the structural changes of elastic fibers by histological procedures. However, the thermally denatured collagen affects the elastic staining and made the analysis of elastic fiber structural alternations impossible. Judging from the serial images obtained at different temperatures, our results suggest that the small autofluorescent aggregates originate from the disrupted elastic fibers.

We also attempted to quantify our observations. Our approach is to calculate the average SHG and AF intensities across the entire large area image at each temperature. The temperature dependence of SHG and AF signals is shown in Fig. 3. The plots show an interesting trend. It is notable that the AF only fluctuates with increasing temperature [Fig. 3(a)]. This result is consistent with the AF images showing that collagen can still be traced by its AF even at high temperatures. On the other hand, the SHG signal shows a different trend. At sufficiently high temperatures, the SHG signal tends to decrease [Fig. 3(b)].

However, since the fiber distribution within the dermis is not homogeneous, the intensity variations shown in Fig. 3 may be due to the fiber distributions and morphological features of the regions being imaged. Therefore, to avoid this artifact, we used the ratiometric definition of AF-to-SHG index of dermis (ASID) of

$$
\mathrm{ASID}=A / S
$$

to characterize the thermal effects on the dermis. In this definition, $A$ is the average AF intensity and $S$ is the average SHG intensity of the images.

In this nomenclature, a highly autofluorescent sample (relative to SHG signal) would have a higher ASID while a specimen containing intense SHG signal (compared with AF) would have a lower ASID. For the thermally treated skin specimens, we computed the ASID from the average AF and SHG intensities obtained across the entire image and the results are plotted in Fig. 4. At temperatures lower than $60^{\circ} \mathrm{C}$, the ASID is almost constant. This result also solidifies our use of a ratiometric approach in characterizing the thermal damage of the dermis in that the ratiometric analysis is not affected by the inhomogeneous fiber distribution. As the plot shows, the ASID starts to increase at $60^{\circ} \mathrm{C}$ and a rising trend of ASID is observed when the temperature is further elevated. This index is more sensitive in predicting dermal thermal damage as compared with the SHG intensities alone.

\section{Discussion}

Our results support the fact that qualitative and quantitative multiphoton AF and SHG microscopy is effective in identifying the extent of human dermal thermal damage. Consistent with our previous reports on rat tail tendons, ${ }^{22}$ we observed that at sufficiently high temperatures, the collagen fiber structures in the dermis become disrupted, corresponding to a decrease in the SHG intensity. Compared with rat tail tendons, the inhomogeneous composition of dermal structures can make quantitative analysis difficult. The isolated SHG intensity can fluctuate before the denaturation temperature and complicates the analysis attempts. We observed a parallel change of SHG intensity and AF of the dermis before the denaturation temperature of $60^{\circ} \mathrm{C}$. This indicates that the fluctuation of AF and SHG intensity can be accounted for by the inhomogeneous distribution of fibers in the regions imaged. However, when a ratiometric approach utilizing ASID is used for analysis, this index is shown to be able to better characterize the degree of dermal thermal damage. We found that AF can serve as a reference to compensate for the inhomogeneous distribution of fibers in the selected regions. Hence, this index is very useful in analysis of the relatively inhomogeneous structures such as dermis. 


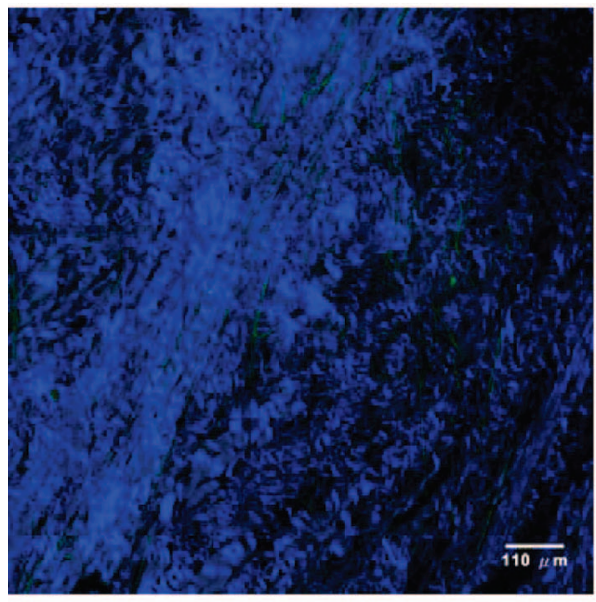

(a) $25^{\circ} \mathrm{C}$

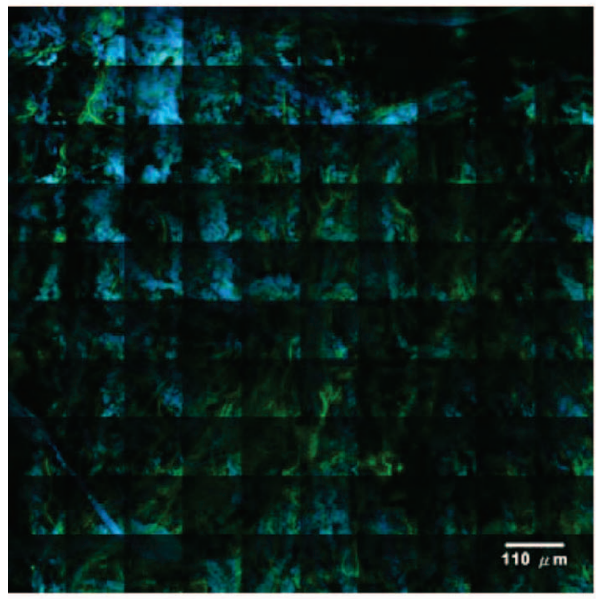

(c) $60^{\circ} \mathrm{C}$

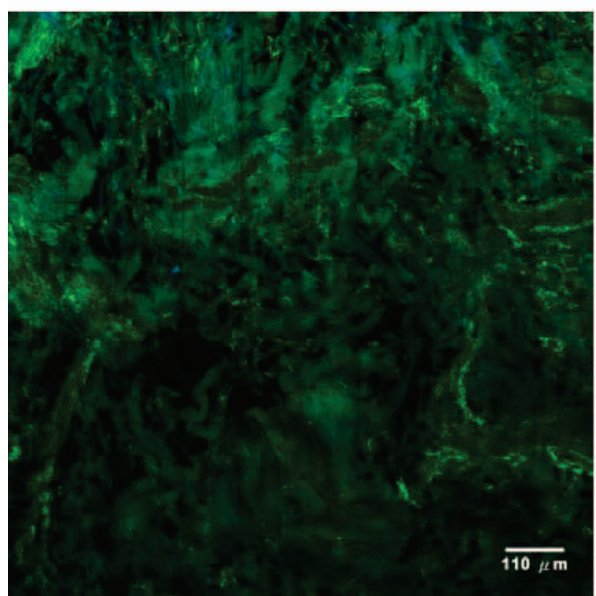

(e) $80^{\circ} \mathrm{C}$

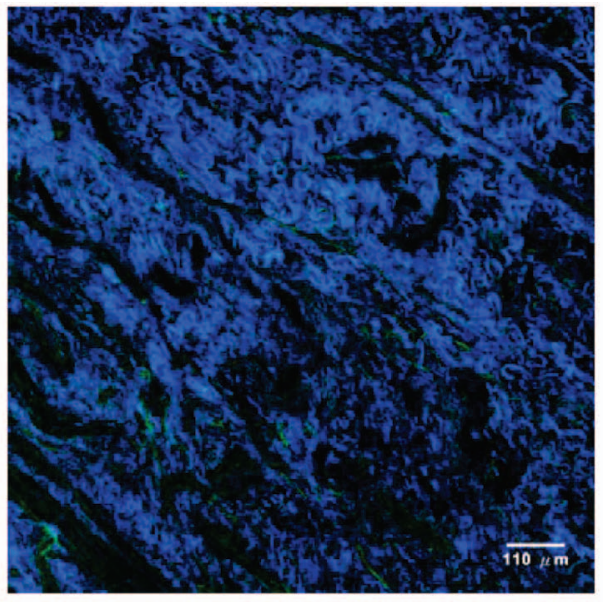

(b) $55^{\circ} \mathrm{C}$

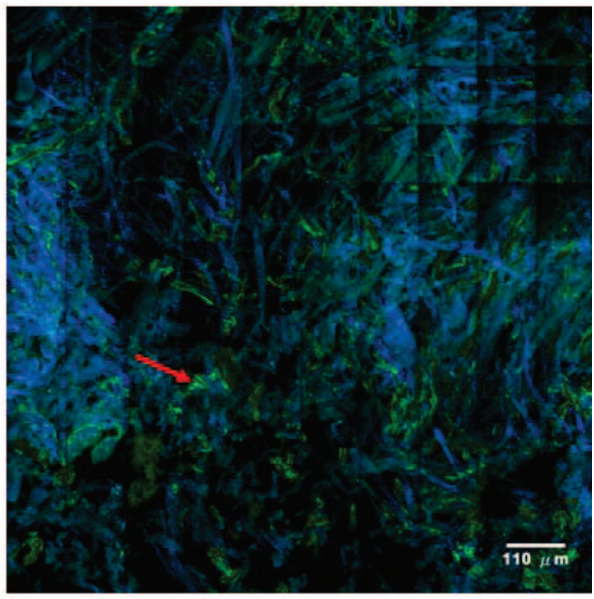

(d) $65^{\circ} \mathrm{C}$

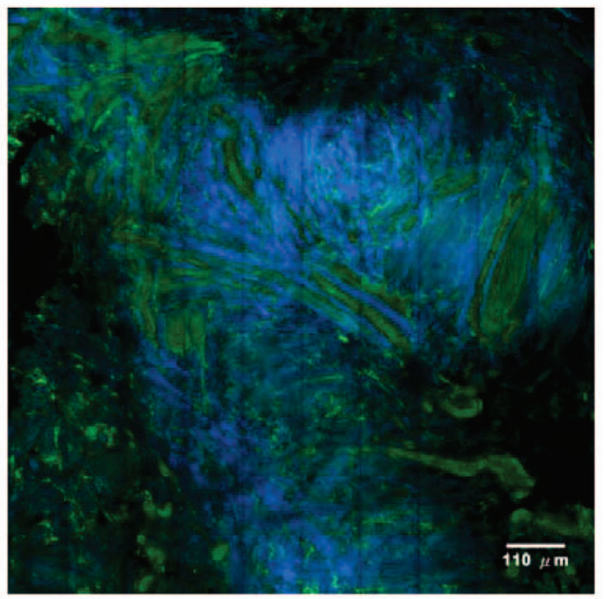

(f) $95^{\circ} \mathrm{C}$

Fig. 2 Combined AF/SHG images of dermal structures treated thermally at 25, 55, 60, 65, 80, and $95^{\circ} \mathrm{C}$ (bars $110 \mu \mathrm{m}$ ). SHG signals are blue and $\mathrm{AF}$ signals are green. 

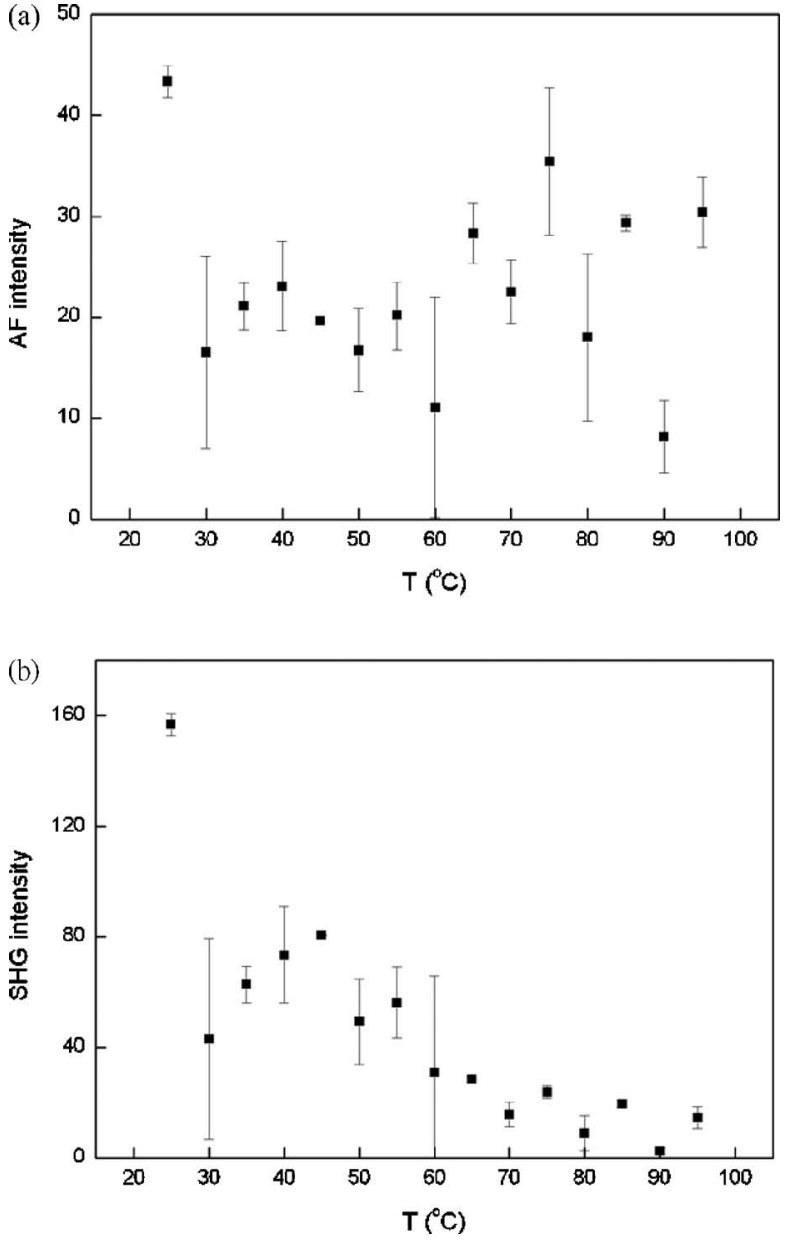

Fig. 3 Dependence of (a) autofluorescence intensity and (b) SHG intensity on temperatures ranging from 25 to $95^{\circ} \mathrm{C}$ (error bars indicate calculated standard deviations).

The intensity of AF from collagen shows a different trend of changes associated with thermal treatment. Since SHG is sensitive to the three-dimensional packing of collagen molecules, the disruption of SHG at $60^{\circ} \mathrm{C}$ and higher temperature indicates that collagen structures responsible for SHG are disrupted. It is suggested that thermal treatment breaks the hydrogen bonds that stabilize the native triple helical molecular structure of collagen, leading to an irreversible transformation of collagen molecules into a random coil. ${ }^{26,27}$ Hence, SHG can be used as a tool to monitor the preservation of original hydrogen bonds in collagen during thermal treatment. However, the $\mathrm{AF}$ of collagen is preserved even at temperatures above $60^{\circ} \mathrm{C}$. It is suggested that collagen $\mathrm{AF}$ is from the cross-linkers of collagen fibers. ${ }^{28}$ Judging from our results, the cross-linkers are less susceptible to the heating process. This is of significance in that after the disruption of SHG signals, AF can still be used to trace the presence of structurally altered collagen. Using AF as a guide, we observed that after the hydrogen bonds are disrupted, collagen tissue in the dermis continues to undergo structural modifications at higher temperature. The fibrous structures are gradually replaced by a molten amorphous structure. In addition to monitoring collagen thermal damage, this can be used to monitor the healing

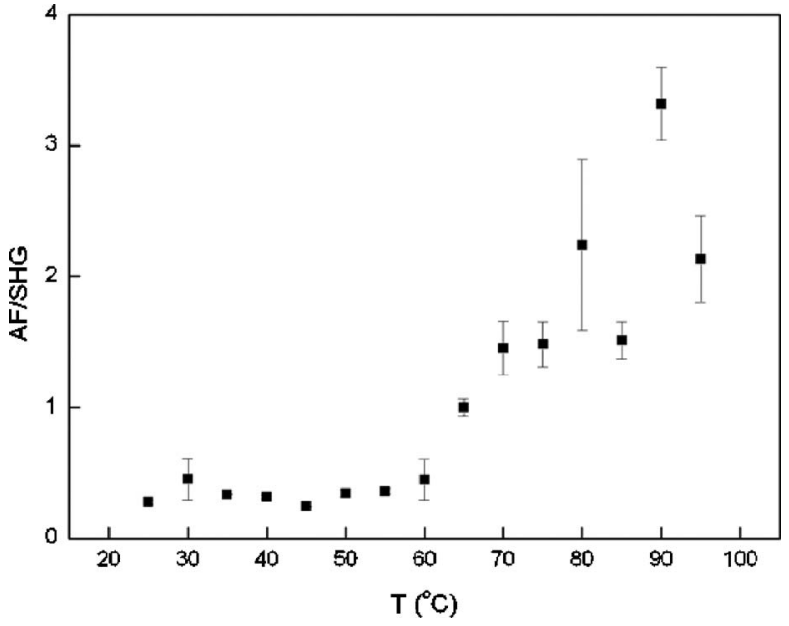

Fig. 4 Dependence of autofluorescence to second-harmonicgeneration index of dermis (ASID) on temperatures (error bars indicate calculated standard deviations).

and remodeling process of collagen after thermal injuries.

In addition, the morphological structures of autofluorescent elastic fibers also change with increasing temperatures. Specifically, we observed that long fine fibrous elastic fibers started to fracture into short fibers and condense into intensely autofluorescent aggregates at $65^{\circ} \mathrm{C}$.

\section{Conclusion}

The thermal damage to dermis can be morphologically and quantitatively analyzed by mulitphoton AF and SHG imaging. Collagen and elastic fibers have different susceptibility to thermal damage and their structural changes respond accordingly. The quantitative changes are confirmed by the analysis of the ASID factor we introduce to characterize the relative changes of AF to SHG intensities. The ASID index can bypass artifacts associated with the inhomogeneous distributions of the dermal fibers and shows that the denaturation temperature of dermal collagen is $60^{\circ} \mathrm{C}$. This study demonstrates that multiphoton microscopy has the potential to be developed into an in vivo diagnostic technique capable of assessing thermal dermal damage and the remodeling process associated with thermal injuries.

\section{Acknowledgment}

Support for this work is provided by the National Research Program for Genomic Medicine of the National Science Council, Taiwan (NSC 94-3112-B-002-015-Y and NSC 933112-B-002-034).

\section{References}

1. P. H. Koster, C. M. van der Horst, M. J. van Gemert, and A. C. van der Wal, "Histologic evaluation of skin damage after overlapping and nonoverlapping flashlamp pumped pulsed dye laser pulses: a study on normal human skin as a model for port wine stains," Lasers Surg. Med. 28, 176-181 (2001).

2. M. Niemz, Laser-Tissue Interactions, Springer-Verlag, Berlin (2002).

3. R. E. Fitzpatrick, M. P. Goldman, N. M. Satur, and W. D. Tope, "Pulsed carbon dioxide laser resurfacing of photoaged facial skin," Arch. Dermatol. 132, 395-402 (1996). 
4. K. A. Khatri, E. V. Ross, J. M. Grevelink, C. M. Magro, and R. R. Anderson, "Comparison of erbium: YAG and carbon dioxide lasers in resurfacing of facial rhytides," Arch. Dermatol. 135, 391-397 (1999).

5. D. J. Goldberg, "Full-face nonablative dermal remodeling with a 1320 nm Nd:YAG laser," Dermatol. Surg. 26, 915-918 (2000).

6. D. J. Goldberg and K. B. Cutler, "Nonablative treatment of rhytides with intense pulsed light," Lasers Surg. Med. 26, 196-200 (2000).

7. R. Fitzpatrick, R. Geronemus, D. Goldberg, M. Kaminer, S. Kilmer, and J. Ruiz-Esparza, "Multicenter study of noninvasive radiofrequency for periorbital tissue tightening," Lasers Surg. Med. 33, 232242 (2003).

8. L. T. Vo, P. Anikijenko, W. J. McLaren, P. M. Delaney, D. H. Barkla, and R. G. King, "Autofluorescence of skin burns detected by fiberoptic confocal imaging: evidence that cool water treatment limits progressive thermal damage in anesthetized hairless mice," J. Trauma 51, 98-104 (2001).

9. L. T. Vo, G. D. Papworth, P. M. Delaney, D. H. Barkla, and R. G. King, "A study of vascular response to thermal injury on hairless mice by fibre optic confocal imaging, laser doppler flowmetry and conventional histology," Burns 24, 319-324 (1998).

10. R. L. Sheridan, K. T. Schomaker, L. C. Lucchina, J. Hurley, L. M Yin, R. G. Tompkins, M. Jerath, A. Torri, K. W. Greaves, and D. P. Bua, "Burn depth estimation by use of indocyanine green fluorescence: initial human trial," J. Burn Care Rehabil. 16, 602-604 (1995).

11. B. H. Park, C. Saxer, S. M. Srinivas, J. S. Nelson, and J. F. de Boer, "In vivo burn depth determination by high-speed fiber-based polarization sensitive optical coherence tomography," J. Biomed. Opt. 6, 474-479 (2001).

12. Z. B. Ziazi, T. J. Essex, R. Papini, D. Scott, N. R. Mclean, and M. J. Black, "New laser-doppler scanner, a valuable adjunct in burn depth assessment," Burns 19, 485-489 (1993).

13. A. T. Yeh, B. Kao, W. G. Jung, Z. Chen, J. S. Nelson, and B. J. Tromberg, "Imaging wound healing using optical coherence tomography and multiphoton microscopy in an in vitro skin-equivalent tissue model," J. Biomed. Opt. 9, 248-253 (2004).

14. K. Konig and I. Riemann, "High-resolution multiphoton tomography of human skin with subcellular spatial resolution and picosecond time resolution," J. Biomed. Opt. 8, 432-439 (2003).

15. Y. Sun, J. W. Su, W. Lo, S. J. Lin, S. H. Jee, and C. Y. Dong, "Multiphoton polarization imaging of the stratum corneum and the dermis in ex-vivo human skin," Opt. Express 11, 3377-3384 (2003).

16. W. Denk, J. H. Strickler, and W. W. Webb, "Two-photon laser scanning fluorescence microscopy," Science 248, 73-76 (1990).
17. P. T. C. So, C. Y. Dong, B. R. Masters, and K. M. Berland, "Twophoton excitation fluorescence microscopy," Annu. Rev. Biomed. Eng. 2, 399-429 (2000).

18. S. J. Lin, R. J. Wu, H. Y. Tan, W. Lo, W. C. Lin, T. H. Young, C. J Hsu, J. S. Chen, S. H. Jee, and C. Y. Dong, "Evaluating cutaneous photoaging by use of multiphoton fluorescence and second harmonic generation microscopy," Opt. Lett. 30, 2275-2277 (2005).

19. A. Zoumi, A. Yeh, and B. J. Tromberg, "Imaging cells and extracellular matrix in vivo by using second-harmonic generation and twophoton excited fluorescence," Proc. Natl. Acad. Sci. U.S.A. 99, 11014-11019 (2002).

20. W. R. Zipfel, R. M. Williams, R. Christie, A. Y. Nikitin, B. T. Hyman, and W. W. Webb, "Live tissue intrinsic emission microscopy using multiphoton-excited native fluorescence and second harmonic generation," Proc. Natl. Acad. Sci. U.S.A. 100, 7075-7080 (2003).

21. P. J. Campagnola and L. M. Loew, "Second-harmonic imaging microscopy for visualizing biomolecular arrays in cells, tissues and organisms," Nat. Biotechnol. 21, 1356-1360 (2003).

22. S. J. Lin, C. Y. Hsiao, Y. Sun, W. Lo, W. C. Lin, G. J. Jan, S. H. Jee, and C. Y. Dong, "Monitoring the thermally induced structural transitions of collagen using second harmonic generation microscopy," Opt. Lett. 30, 622-624 (2005).

23. S. J. Lin, W. Lo, H. Y. Tan, J. Y. Chan, W. L. Chen, S. H. Wang, Y. Sun, W. C. Lin, J. S. Chen, C. J. Hsu, J. W. Tjiu, H. S. Yu, S. H. Jee, and C. Y. Dong, "Prediction of heat-induced collagen shrinkage by use of second harmonic generation microscopy," J. Biomed. Opt. 11, 034020 (2006)

24. M. Han, G. Giese, and J. F. Bille, "Second harmonic generation imaging of collagen fibrils in cornea and sclera," Opt. Express 13(15), 5791-5797 (2005).

25. R. M. Williams, W. R. Zipfel, and W. W. Webb, "Interpreting secondharmonic generation images of collagen I fibrils," Biophys. J. 88(2), 1377-1386 (2005)

26. N. T. Wright and J. D. Humphrey, "Denaturation of collagen via heating: an irreversible rate process," Апnи. Rev. Biomed. Eng. 4 109-128 (2002).

27. K. Hayashi, G. Thabit III, J. J. Bogdanske, L. N. Mascio, and M. D Markel, "The effect of nonablative laser energy on the ultrastructure of joint capsular collagen," Arthroscopy 12, 474-481 (1996).

28. N. Kollias, R. Gillies, M. Moran, I. E. Kochevar, and R. R. Anderson, "Endogeneous skin fluorescence includes bands that may serve as quantitative markers of aging and photoaging," J. Invest. Dermatol. 111, 776-780 (1998). 